\title{
Do Various Glitazones Have the Same Risk of Acute Myocardial Infarction? Indirect Evidence from a Population-Based Norwegian Cohort Study
}

\author{
Ivar Aursnes $^{*}, 1$, Marianne Klemp ${ }^{1}$ and Til Stürmer ${ }^{2}$ \\ ${ }^{I}$ Department of Pharmacology, University of Oslo, Oslo, Norway \\ ${ }^{2}$ Department of Epidemiology, University of North Carolina Gillings School of Global Public Health, Chapel Hill, NC, \\ USA
}

\begin{abstract}
Objective: Pioglitazone lowers triglycerides and is indifferent towards low-density lipoproteins (LDL), while rosiglitazone has no effect on triglycerides and increases LDL. Our purpose was to test the hypothesis that the risk of acute myocardial infarction (AMI) in thiazolidinediones differs.
\end{abstract}

\begin{abstract}
Methods: We followed a cohort from the Norwegian Prescription Database consisting of 4,009 and 740 first time users of pioglitazone and rosiglitazone respectively for three years. We estimated the propensity score for rosiglitazone $v S$ pioglitazone based on age, gender, and the use of 13 drug classes. We used the initiation of platelet aggregation inhibitors, lipid lowering drugs, beta-adrenergic blockers, and renin-angiotensin inhibitors as a proxy for AMI after testing the validity of these endpoints in a separate cohort of patients suffering their first AMI. We estimated hazard ratios (HR, rosiglitazone vs pioglitazone) and their 95 percent confidence intervals (CI) for AMI using Cox proportional hazards models stratified by propensity score deciles.
\end{abstract}

Results: During the first six months after initiation the incidences of the initiation of platelet aggregation inhibitors were the same with both glitazones ( $\mathrm{HR}=1.0 ; 95 \% \mathrm{CI}$ : 0.65-1.52). More than six months after initiation, rosiglitazone was associated with an increased risk of initiating platelet aggregation inhibitors compared with pioglitazone $(\mathrm{HR}=1.68 ; 95 \%$ CI: 1.09-2.61). We observed no difference between the glitazones and the initiation of any of the other drug classes assessed.

Conclusions: Albeit indirectly, our cohort study supports the hypothesis that the two thiazolidinediones differ in their risk of AMI, based on monitoring over a period of three years the initiation of drug classes indicated after AMI.

Keywords: Cohort study, propensity score, myocardial infarction, glitazones, diabetes, pharmacoepidemiology.

\section{INTRODUCTION}

Two worldwide registered thiazolidinediones [1, 2], rosiglitazone and pioglitazone, both target insulin resistance through their actions as peroxisome-proliferator-activated receptor- $\gamma$ agonists. Although quite similar in their effect on glucose metabolism, the two glitazones differ in their effect on lipid modification. Pioglitazone lowers triglycerides and is indifferent towards low density lipoproteins (LDL), whereas rosiglitazone has no effect on triglycerides but increases LDL [3]. It is not known whether these differences lead to different effects on cardiovascular complications when used in patients with type 2 diabetes because no headto-head long-term evaluation of the two drugs has been undertaken. Moreover, to our knowledge no such study is on its way. In that case one has to resort to observational data. In Norway, we have accurate but incomplete data detailing the use of thiazolidinediones in diabetic patients. Although we do not gain information directly on patients' morbidity, like frequency of hospitalization and diagnoses, valuable

*Address correspondence to this author at the Department of Pharmacology, University of Oslo, PO Box 1057 Blindern, 0316 Oslo, Norway; Tel: +4722840290; Fax:+4722840202; E-mail: i.a.aursnes@medisin.uio.no indirect information is readily obtained from a recently established prescription based drug register.

Our intention was to prospectively follow a cohort of first-time glitazone users to see whether, as time went by, the patients were prescribed specific cardiovascular drugs as signs of different propensities for cardiovascular complications. During the follow-up it was reported by others that rosiglitazone users fared worse than patients taking a placebo [4]. As a consequence we ended our study in May 2007 because we thought this information would influence the use of the drug to an extent that would disturb the outcome of our study. We have since learned that it had been suspected for several years that rosiglitazone is associated with other types of heart disease besides heart failure. A higher frequency of heart failure is a recognized adverse effect of all glitazones [5].

Although the use of pioglitazone is similarly associated with a risk of fluid retention and heart failure [6], no reports have come to light that connect the drug with a higher frequency of other heart diseases, like coronary disease. In fact, a recent meta-analysis of randomized clinical trials indicates that pioglitazone is associated with a lower incidence of coronary heart disease when compared with a placebo [7]. Thus our purpose was to find out whether the 
different outcomes of trials on the two drugs are reflected in the records of the drug prescription database. Our prespecified hypothesis was that we would obtain indications that patients on pioglitazone had a better prognosis compared with rosiglitazone i.e. less indications of newly diagnosed coronary disease. We also expected that this difference would appear after some delay, as coronary atheromatosis is a slow growing process.

\section{RESEARCH DESIGN AND METHODS}

\section{Study Subjects}

Since Jan. 1, 2004, all prescription drugs dispensed through pharmacies, the only way to obtain drugs outside of health institutions in Norway, have been registered in the Norwegian Prescription Database (NorPD) and linked to a pseudonym derived from the patient's social security number. At the pharmacies an item number is attached to each dispensed drug. This refers to the ATC (Anatomical Therapeutic Chemical) number given to the drug when registered by the Norwegian Medicinal Agency (Legemiddelverket). The NorPD uses the ATC code [8]. The seven-digit ATC classification has a hierarchical five-level structure starting with the broad indication (e.g. "A" for "Alimentary tract and metabolism" [9]) and ending with a specific chemical substance. There were two drugs registered as glitazones (thiazolidinediones), ATC-code: A10BG, rosiglitazone (Avandia, GSK, ATC-code: A10BG02) and pioglitazone (Actos, Takeda, ATC-code: A10BG03). Both drugs were first registered in Norway in 2000.

We obtained records of all first time prescriptions for glitazones dispensed between July 1, 2004 and Dec. 31, 2005. Patients were regarded as incident users of glitazones if they had been without a glitazone prescription for at least six months. In practice most of the users were using the drug for the very first time. The first dispensing date was defined as the cohort entry date or baseline. After discarding 6.5 percent of the glitazone prescriptions with missing personal identifiers, we obtained a cohort of 4,766 individuals and collected records of all prescriptions dispensed to these patients between Jan. 1, 2004 and April 30, 2007.

As of October 2004, the patients were reimbursed most of the costs of the two drugs by Social Security Services. By regulation, one prescription provides a three-month supply of the drug. We therefore calculated time on the drug as the number of days from the first prescription (baseline) to the end of treatment (120 days after the last prescription of the drug, pragmatically chosen to allow for an additional 30 days after the assumed end of the last prescription), switching to another glitazone, death, or end of the study (April 30, 2007), whichever came first.

With the help of the defined daily doses for rosiglitazone (6 $\mathrm{mg}$ ) and pioglitazone $(30 \mathrm{mg})$, we calculated from the database that the prescriptions' average coverage was 88 percent (SD $42 \%$ ) of a defined daily dose for each patient per day from the baseline until 90 days after the last prescription.

\section{Outcome Measures}

In the absence of clinical data, we defined our endpoints as the initiation of specific drug classes that are indicated after an acute myocardial infarction (AMI). We tested the validity of these endpoints in a separate validation study consisting of a cohort of 170 consecutive patients admitted to Lovisenberg Hospital in Oslo between Jan. 1, 2005 and Oct. 30, 2006 and discharged with the diagnosis of a first time AMI in accordance with the specific ICD 10 coding for this condition: I21. We collected prescriptions dispensed between July 1, 2004 and April 30, 2007, and registered the consumption of warfarin (ATC-code: B01AA), platelet aggregation inhibitors (B01AC), beta-adrenergic blockers (C07), renin-angiotensin antagonists (C09), and lipid modifying drugs $(\mathrm{C} 10)$ during a period starting six months before the date of hospitalisation and ending six months after. According to current guidelines in Norway the above drug classes are indicated in patients after an AMI and are thus routinely used in this setting. Of course, platelet aggregation inhibitors and lipid modifying drugs are also used extensively in patients without a diagnosis of AMI [10]. A special feature of the platelet aggregation inhibitor acetylsalicylic acid (aspirin) is that in Norway the low dose enterosoluble form is available by physician's prescription only, making the NorPD especially apt for our present purpose. It is also notable that in the glitazone cohort, aspirin constituted 91 percent of the prescriptions in the group. The rest referred to clopidogrel, ticlopidine, and dipyridamole.

The validation study had no control group and our inferences were made from drug initiations coinciding with the time of diagnosis of AMI. After assessing the association between AMI and the initiation of each of these drug classes in the validation study, we then addressed the initiation of each of these five different drug classes as a separate outcome in our main cohort of glitazone users, provided that the glitazone initiators had not received similar drugs during the six months prior to the baseline.

\section{Covariates}

The NorPD database contains the patient's year of birth and gender, as well as the prescribing physician's year of birth, gender, and speciality (internist yes/no, general practitioner yes/no). Over time several physicians can prescribe to the same patient. We only recorded the speciality of the first prescriber responsible for the choice of glitazone. As proxy for comorbidity we registered any use of the following drug groups during the six months before baseline: insulin (A10A), oral antidiabetics (A10B), warfarin (B01AA), platelet aggregation inhibitors (B01AC), digitalis (C01AA), antiarrythmics (C01B), antihypertensives (C02), low ceiling diuretics $(\mathrm{C} 03 \mathrm{~A})$, high ceiling diuretics $(\mathrm{C} 03 \mathrm{C})$, potassium sparing diuretics $(\mathrm{C} 03 \mathrm{D}+\mathrm{E})$, beta-adrenergic blockers (C07), calcium antagonists (C08), renin-angiotensin inhibitors (C09), and lipid lowering drugs (C10). Any prescription filled during the six months period prior to the baseline was coded as use of the corresponding drug class. All others were classified as non-use. This coding is common in administrative databases and results in no missing values for these covariates.

\section{Statistical Analyses}

Since the use of antiarrythmics occurred only in the rosiglitazone group, we omitted this variable along with the data of the 15 patients using antiarrhytmics from further analyses. In order to adjust for possible confounders in the 
Table 1. Exposure Group Characteristics Including Percentage of Users of Various Drugs During the Last Six Months Before Baseline

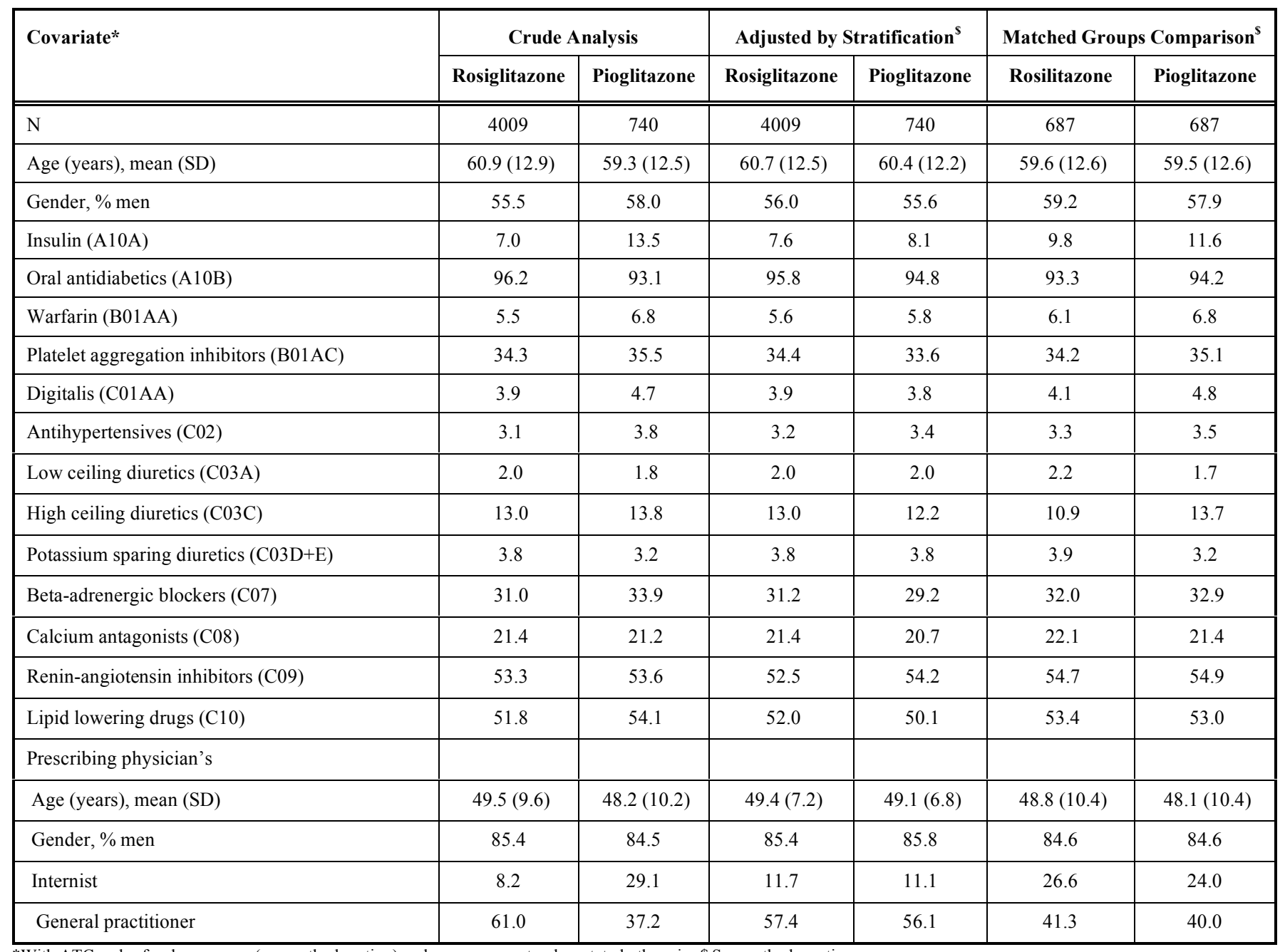

*With ATC codes for drug groups (see method section), values are percent unless stated otherwise $\$$ See methods section.

material, we used logistic regression and the remaining 19 variables to estimate the propensity to initiate pioglitazone $v \mathrm{~s}$ the initiation of rosiglitazone. The propensity score is a summary score that helps to balance observed covariates between two treatment groups and thus control for confounding by these observed covariates $[11,12]$. We used these propensity scores to stratify the study population into 10 groups of equal size (deciles). For binary variables we cross tabulated deciles groups $v s$ each variable and separately for the two glitazone exposure groups, and calculated the percentage of users in each cell. The adjusted distributions of variables between the exposure groups could then be calculated as the sum of the cell percentages divided by 10 . For age we similarly obtained weighted averages after tabulating deciles groups against means and the standard deviations for the variable. We also matched each patient initiating pioglitazone to one patient initiating rosiglitazone. The patients were chosen at random without replacement within one standard error of the linear variant of the propensity score using a standard Microsoft Excel routine [13].

We calculated the incidence rates for each endpoint and exposure based on the number of events and the person years of exposure. We fitted a Cox proportional hazard model stratified on propensity score deciles with only the exposure group as a covariate and recorded the hazard ratios with their 95 percent confidence intervals. Similarly, we modelled the matched groups and illustrated survival until the initiation of each outcome with life table diagrams with the help of SPSS, version 14.0. We used the matched groups mainly for illustrative purposes, while the stratification procedure allowed us to include all data and thereby obtain maximum statistical power.

Correspondingly, in the validation study we used the same software (technically a life table procedure) to analyse the use of the above mentioned drugs in patients six months before and after an AMI.

\section{RESULTS}

The distribution of covariates between users of rosiglitazone and pioglitazone is shown for the whole cohort in the first two columns of Table 1. Columns 3 and 4 show that the stratification of the propensity scores had marked effects on the distribution of variables between the two exposure groups. Notably, for the first prescribing physician, 


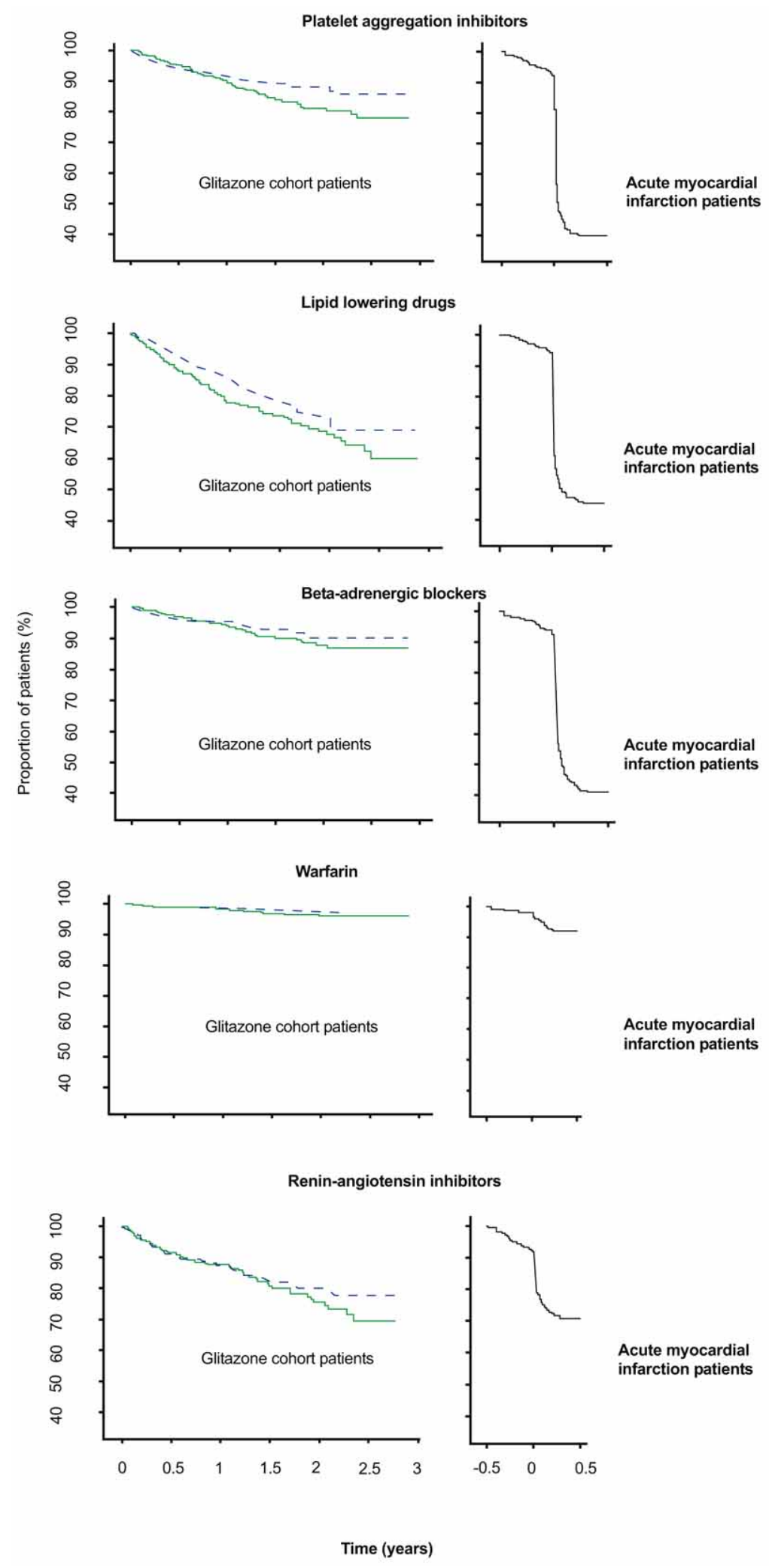

Fig. (1). Proportions of patients without various first uses of various drug groups.

Matched subgroups of a cohort of patients initiating rosiglitazone (green, continuous) or pioglitazone (blue, stippled), with corresponding curves for drug uses in patients six months before and after an acute myocardial infarction.

the large differences in specialities are almost obliterated. Also, the use of insulin is very similar for the two groups after the adjustment. Similar effects can be seen in Columns
5 and 6 after matching the two exposure groups on the propensity score. 
The outcome of the study is illustrated for the various index drugs in Fig. (1) by comparing the results for matched study groups on the left with corresponding new drug initiations in the validation study on the right. The difference between the two curves on the left is the observed effect measure distinguishing the two groups of patients in the glitazone cohort. For the patients in the validation study that we knew had an acute myocardial infarction, the point of interest is the difference in the slope of the curves before and after the date of hospitalization (day zero). We did not quantify the latter difference but it is readily observed from the diagrams that there is a steep increase in use of platelet aggregation inhibitors, lipid lowering drugs, and betaadrenergic blockers after myocardial infarction.

Moreover, it can also be observed that divergence of the two glitazone curves occurs after a delay, mostly about six months after baseline. One can observe that when there is little extra use of a drug following an AMI (Fig. 1D-E), there is hardly any divergence.

Numerical expressions of divergences are given in Table $\mathbf{2}$ for unadjusted groups, for the matched groups that we used for constructing the diagrams, and for the treatment groups that we had adjusted for possible confounding variables by means of stratification. When we included all study subjects in the stratification procedure we obtained the highest statistical power.
During the first six months after the initiation of glitazones, there was no difference in the incidence of initiation of platelet aggregation inhibitors between the cohorts (Fig. 1A and Table 2). After six months, however, the incidence of platelet aggregation inhibition was greater in patients on rosiglitazone compared with patients on pioglitazone $(\mathrm{HR}=1.68 ; 95 \% \mathrm{CI}$ : 1.09-2.61), and there was also a difference in initiators of lipid lowering drugs (Fig. 1B and Table 2). There were few initiators of beta-adrenergic blockers in both cohorts with almost no difference between the two glitazones (Fig. 1C and Table 2). Other differences between the groups in the cohort were not striking. Warfarin was seldom used either in the cohort or after myocardial infarction, and the use of renin-angiotensin inhibitors was similar in the two groups (Fig. 1D, $\mathbf{E}$ and Table 2).

According to observations in the validation study (Fig. 1A-E), the initiation of platelet aggregation inhibitors, lipid lowering drugs, and beta-adrenergic blockers would be expected to be an indicator of AMI. In the validation study the mean age of the control patients was 66.4 years (SD 14.9) and 64 percent were men. The life table diagrams for the patients show that platelet aggregation inhibitors, betaadrenergic blockers, and lipid lowering drugs all show a marked increase in initiation in the first days after a first time myocardial infarction, whereas warfarin and reninangiotensin inhibitors are little used for this indication in Norway today (Fig. 1D-E).

Table 2. Outcomes with Various Times Until the First Use of Drug as Endpoints

\begin{tabular}{|c|c|c|c|c|c|c|c|c|c|c|c|}
\hline \multirow[b]{2}{*}{$\begin{array}{c}\text { Drug Group } \\
\text { and Exposure } \\
\text { Period for } \\
\text { Hazard Ratio } \\
\text { Calculations, } \\
\text { Days After } \\
\text { Baseline }\end{array}$} & \multicolumn{4}{|c|}{ Rosiglitazone } & \multicolumn{4}{|c|}{ Pioglitazone } & \multicolumn{3}{|c|}{$\begin{array}{c}\text { Hazard Ratio* (95\% Confidence } \\
\text { Intervals) }\end{array}$} \\
\hline & $\mathbf{n}$ & $\begin{array}{l}\text { Person } \\
\text { Years }\end{array}$ & $\begin{array}{l}\text { No. of } \\
\text { Events }\end{array}$ & $\begin{array}{c}\text { Incidence } \\
\text { Rate/100 } \\
\text { 0 Person } \\
\text { Years }\end{array}$ & $\mathbf{n}$ & $\begin{array}{l}\text { Person } \\
\text { Years }\end{array}$ & No. of Events & $\begin{array}{c}\text { Incidence } \\
\text { Rate/1000 } \\
\text { Person Years }\end{array}$ & Crude & Matched & Stratified \\
\hline \multicolumn{12}{|c|}{ Platelet aggregation inhibitors } \\
\hline $1-180$ & 585 & 1221 & 143 & 117 & 125 & 216.6 & 29 & 134 & $\begin{array}{c}0.99 \\
(0.67-1.48)\end{array}$ & $\begin{array}{c}0.75 \\
(0.42-1.34)\end{array}$ & $\begin{array}{c}1.00 \\
(0.65-1.52)\end{array}$ \\
\hline $181-1100$ & 2049 & 3352 & 259 & 77 & 352 & 571.2 & 23 & 40 & $\begin{array}{c}1.93 \\
(1.26-2.95)\end{array}$ & $\begin{array}{c}2.30 \\
(1.33-2.99)\end{array}$ & $\begin{array}{c}1.68 \\
(1.09-2.61)\end{array}$ \\
\hline $1-1100$ & 2634 & 3549 & 402 & 113 & 477 & 611.8 & 52 & 85 & $\begin{array}{c}1.34 \\
(1.00-1.79)\end{array}$ & $\begin{array}{c}1.35 \\
(0.92-1.98)\end{array}$ & $\begin{array}{c}1.20 \\
(0.89-1.61)\end{array}$ \\
\hline \multicolumn{12}{|c|}{ Lipid lowering drugs } \\
\hline $1-1100$ & 1932 & 2363 & 473 & 200 & 340 & 404.0 & 68 & 168 & $\begin{array}{c}1.19 \\
(0.93-1.54)\end{array}$ & $\begin{array}{c}1.30 \\
(0.93-1.80)\end{array}$ & $\begin{array}{c}1.27 \\
(0.98-1.66)\end{array}$ \\
\hline \multicolumn{12}{|c|}{ Beta-adrenergic blockers } \\
\hline $1-1100$ & 2766 & 3986 & 186 & 47 & 489 & 655.1 & 37 & 56 & $\begin{array}{c}0.84 \\
(0.589- \\
1.193)\end{array}$ & $\begin{array}{c}1.19 \\
(0.749- \\
1.883)\end{array}$ & $\begin{array}{c}0.81 \\
(0.56-1.18)\end{array}$ \\
\hline \multicolumn{12}{|l|}{ Warfarin } \\
\hline $1-1100$ & 3788 & 5687 & 83 & 15 & 690 & 982.9 & 15 & 15 & $\begin{array}{c}0.96 \\
(0.55-1.66)\end{array}$ & $\begin{array}{c}1.19 \\
(0.59-2.41)\end{array}$ & $\begin{array}{c}0.84 \\
(0.47-1.50)\end{array}$ \\
\hline \multicolumn{12}{|c|}{ Renin-angiotensin inhibitors } \\
\hline $1-1100$ & 1871 & 2436 & 316 & 130 & 330 & 409.4 & 52 & 127 & $\begin{array}{c}1.03 \\
(0.77-1.38)\end{array}$ & $\begin{array}{c}1.14 \\
(0.78-1.67)\end{array}$ & $\begin{array}{c}1.04 \\
(0.76-1.41)\end{array}$ \\
\hline
\end{tabular}

*Proportional hazards model regression matched 1:1 on propensity scores or stratified by deciles of propensity scores (see methods section). 


\section{DISCUSSION}

We found a difference between the two glitazones in the incidence of platelet aggregation inhibitors used six months after the initiation of glitazone. In Norway, consumption in this drug group consists mainly of acetyl-salicylic acid (aspirin) and the anti-thrombotic dosage is defined as 75 $160 \mathrm{mg}$ daily. The most popular product comes from a local manufacturer, is the only aspirin product registered under $\mathrm{B} 01 \mathrm{AC}$, and is restricted by a prescription requirement, while higher doses of aspirin are sold over the counter and registered as analgesics. Platelet aggregation inhibitors are systematically dispensed to patients in a post-myocardial infarction situation. According to Norwegian wholesale statistics 64 defined daily doses (DDD) are consumed per 1,000 inhabitants per day. This extensive drug use is confirmed by NorPD, which shows that 193 per 1,000 inhabitants between 60 and 69 years of age received at least one prescription for this type of drug during 2006 [14]. The total use of drugs in the group B01AC was 198 DDDs per 1,000 inhabitants per day.

There was not a definite increase of consumption of the other post-myocardial infarction drugs in the rosiglitazone group. We will therefore not conclude that our findings indicate a substantial degree of increased incidence of myocardial infarction as such, rather that infarctions as well as other signs of coronary disease like unstable angina on a whole were diagnosed more frequently in the rosiglitazone group.

There are limitations to our use of the validation study. The patients diagnosed with an AMI came from one hospital only, whereas those in the glitazone cohort came from all of Norway. The AMI patients were also about five years older and included a somewhat higher percentage of men. However, the findings are in keeping with the recommended use of post myocardial infarction medication and in accordance with current Norwegian guidelines [15].

Our findings coincide with the idea expressed in a recent publication saying that the effects of rosiglitazone on the risk for myocardial infarction and cardiovascular death are uncertain [16]. Our finding that the initiation of betaadrenergic blockers was the same in the two groups may be an indication that there was no difference in the incidence of AMI leading to heart failure. Probably the absolute risk in the cohort with an average age of 60 years is too low to see a substantial number of AMIs during this relatively short follow-up period. However, if the degree of difference in the use of the MI-preventive drugs platelet aggregation inhibitors and lipid lowering drugs is correct, the consequences of choosing one glitazone before the other might be perilous.

Our study has several limitations. Notably, the sensitivity and specificity of our proxy for acute MI is unknown. Results from the validation study indicate that the sensitivity may be acceptable, but the specificity could not be assessed and is certainly low given that many of these drugs are also prescribed for other indications. A low specificity would bias our results towards the null. This needs to be taken into account when interpreting our findings.
We were able to control for only very few covariates, mainly based on drug use as a proxy for co-morbidity. We were unable to control for clinical status including $\mathrm{HbAlc}$ levels, body mass index, and pre-existence of diabetes complications. This means that residual confounding and uncontrolled confounding cannot be ruled out. However, comparisons within a drug class such as thiazolidinediones are probably less prone to confounding, including confounding by indication. We stopped follow-ups at the time of the first reports on a possible association between rosiglitazone and AMI. Interestingly, rosiglitazone was much more prescribed by general practitioners than by specialized internists. The most plausible explanation for this finding is differential marketing.

We do not know whether the dispensed drugs were actually consumed. However, there is a dispensing fee of 36 percent up to a limit of 40 Euro and the large majority of our cohort $(90.2 \%)$ received more than one prescription. Refilling a prescription without actually taking the drug does not make sense, and is therefore unlikely to be a relevant problem in this setting. We censored patients when they stopped refilling their prescriptions and the average coverage was 88 percent of a defined daily dose for each patient per day from the baseline until 90 days after the last prescription.

Owing to its population-based design, the external validity of our findings to the Norwegian population is as a whole determined by design. Indications or the propensity for treatment depending on cardiovascular (CV) risk factors including age can vary between countries, and whether or not our results can be generalized to other populations depends on the assumption that such $\mathrm{CV}$ risk factors affect the effects of the drugs.

Since the publication of the controlled clinical trial metaanalysis in May 2007 [4], leading to the cessation of our cohort study, a whole series of reports have been published [17-20]. Most conclusions and comments tend to view the use of rosiglitazone to regulate blood sugar levels in diabetes type 2 patients as involving an unacceptable degree of cardiovascular risk, but, to our knowledge no country has taken decisions to withdraw this drug. Our finding of slight differences between the two thiazolidinediones is disturbing and may possibly move some physicians towards a preference for prescribing pioglitazone. This would be in keeping with the American Diabetes Association (ADA) and the European Association for the Study of Diabetes (EASD) that have issued guidelines that explicitly advise against the use of rosiglitazone for type-2 diabetes [21].

Ethics approval was obtained from the Regional Ethics Committee for Medical Research of East Norway.

\section{ACKNOWLEDGEMENTS}

Dr. Til Stürmer receives investigator-initiated research funding and support as principal investigator from the U.S. National Institute of Aging department of the National Institutes of Health (RO1 AG023178). He does not accept personal compensation of any kind from any pharmaceutical company, though he receives salary support from unrestricted research grants from pharmaceutical companies. 
The other authors have no relevant conflicts of interest to disclose.

\section{REFERENCES}

[1] Yki-Jarvinen H. Thiazolidinediones. N Engl J Med 2004; 351: 1106-18.

[2] Staels B, Fruchart JC. Therapeutic roles of peroxisome proliferatoractivated receptor agonists. Diabetes 2005; 54: 2460-70.

[3] Goldberg RB, Kendall DM, Deeg MA, et al. A comparison of lipid and glycemic effects of pioglitazone and rosiglitazone in patients with type 2 diabetes and dyslipidemia. Diabetes Care 2005; 28: 1547-54.

[4] Nissen SE, Wolski K. Effect of rosiglitazone on the risk of myocardial infarction and death from cardiovascular causes. N Engl J Med 2007; 356: 2457-71.

[5] Mulrow CD, Cornell J, Localio AR. Rosiglitazone: A thunderstorm from scarce and fragile data. Ann Intern Med 2007; 147: 585-7.

[6] Lago RM, Singh PP, Nesto RW. Congestive heart failure and cardiovascular death in patients with prediabetes and type 2 diabetes given thiazolidinediones: a meta-analysis of randomised clinical trials. Lancet 2007; 370: 1129-36.

[7] Lincoff AM, Wolski K, Nicholls SJ, Nissen SE. Pioglitazone and risk of cardiovascular events in patients with type 2 diabetes mellitus: a meta-analysis of randomized trials. JAMA 2007; 298: 1180-8.

[8] WHO. Collaborating Centre for Drug Statistics Methodology 2007.

[9] http://en.wikipedia.org/wiki/ATC_code_A [Accessed: 10 Mar 2009].
[10] Berger JS, Brown DL, Becker RC. Low-dose aspirin in patients with stable cardiovascular disease: A meta-analysis. Am J Med 2008; 121: 43-9.

[11] Rosenbaum PR, Rubin DB. The central role of the propensity score in observational studies for causal effects. Biometrika 1983; 70: 4155 .

[12] D'Agostino RB. Propensity score methods for bias reduction in the comparison of a treatment to a non-randomized control group. Statistics in Medicine 1998; 17: 2265-81.

[13] http://www.chrp.org/propensity [Accessed: 10 Mar 2009].

[14] http://www.reseptregisteret.no/ [Accessed: 10 Mar 2009].

[15] http://www.legemiddelhandboka.no. Accessed 10 Mar 2009.

[16] Diamond GA, Bax L, Kaul S. Uncertain effects of rosiglitazone on the risk for myocardial infarction and cardiovascular death. Ann Intern Med 2007; 147: 578-81.

[17] Singh S, Loke YK, Furberg CD. Long-term risk of cardiovascular Events With Rosiglitazone: A Meta-analysis. JAMA 2007; 298: 1189-95.

[18] Lipscombe LL, Gomes T, Lévesque LE, Hux JE, Juurlink DN, Alter DA. Thiazolidinediones and cardiovascular outcomes in older patients with diabetes. JAMA 2007; 298: 2634-43.

[19] Berlie HD, Kalus JS, Jaber LA. Thiazolidinediones and the risk of edema: A meta-analysis. Diabetes Res Clin Pract 2007; 76: 279-89.

[20] Walker A, Koro C, Landon J. Coronary heart disease outcomes in patients receiving antidiabetic agents in the PharMetrics database 2000-2007. Pharmacoepidemiol Drug Saf 2008; 17(8): 760-8.

[21] Rosiglitazone no longer recommended. Lancet 2008; 372: 1520. 